\title{
AES for multiscale localization modeling in granular media
}

\author{
Qiushi Chen ${ }^{\mathrm{a}}$, José E. Andrade ${ }^{\mathrm{a}, *}$, Esteban Samaniego ${ }^{\mathrm{b}}$ \\ a Mechanical and Civil Engineering, California Institute of Technology, Pasadena, CA 91125, USA \\ ${ }^{\mathrm{b}}$ Civil Engineering School and Grupo de Ciencias de la Tierra y el Ambiente, Universidad de Cuenca, Cuenca, Ecuador
}

\section{A R T I C L E I N F O}

Article history:

Received 11 October 2010

Received in revised form 7 February 2011

Accepted 24 April 2011

Available online 11 May 2011

\section{Keywords:}

Assumed enhanced strain (AES) method

Strong discontinuities

Localization

Multiscale

DEM

Granular media

\begin{abstract}
A B S T R A C T
This work presents a multiscale strong discontinuity approach to tackle key challenges in modeling localization behavior in granular media: accommodation of discontinuities in the kinematic fields, and direct linkage to the underlying grain-scale information. Assumed enhanced strain (AES) concepts are borrowed to enhance elements for post-localization analysis, but are reformulated within a recently-proposed hierarchical multiscale computational framework. Unlike classical AES methods, where material properties are usually constants or assumed to evolve with some arbitrary phenomenological laws, this framework provides a bridge to extract evolutions of key material parameters, such as friction and dilatancy, based on grain scale computational or experimental data. More importantly, the phenomenological softening modulus typically used in AES methods is no longer required. Numerical examples of plane strain compression tests are presented to illustrate the applicability of this method and to analyze its numerical performance.
\end{abstract}

(c) 2011 Elsevier B.V. All rights reserved.

\section{Introduction}

Failure in granular materials may present in various modes, such as diffuse, localized, etc. Many research efforts have been devoted to understand the failure phenomenon in granular materials (e.g. [1,2]). Diffuse failure refers to failure modes with no strain localization pattern, see e.g. some recent work by Darve et al. [3] and Nicot et al. [4], among others. In this paper, we are particularly interested in studying failure in the form of localized deformations, i.e., large strains occurring over a narrow zone. Macroscopic detection of localization phenomena is relatively well understood and its modeling has been cast within the finite element method, e.g. [5-9]. However, advancement of solutions beyond the localization point remains a challenge in computational mechanics. On the one hand, propagation of localization bands necessitates accommodation of discontinuities in some kinematical fields (e.g. displacements or strains) in a deforming body; on the other hand, the underlying grain structures and particle interactions, which are known to govern material responses at the macroscale, are yet to be taken into account when modeling localization behavior in granular materials. This latter point would allow for enhanced modeling capabilities and higher accuracy.

Regarding discontinuities in kinematical fields, there are usually two types identified in the literature [10]: weak discontinuities, which involve a discontinuous deformation gradient; and strong

\footnotetext{
* Corresponding author.

E-mail address: jandrade@caltech.edu (J.E. Andrade).
}

discontinuities, which involve a discontinuous displacement field. Classical approaches typically consider localization bands as weak discontinuities. However, due to the lack of an intrinsic characteristic length scale, classical rate-independent plasticity models have difficulties in resolving material behavior beyond localization and usually experience pathological mesh dependence $[11,12]$.

An alternative way for analyzing localization problem has been proposed in recent years and is predicated on the strong discontinuity concept, see for example [10,13-21], among others. Failure kinematics related to localization bands are approximated by means of discontinuous displacement fields embedded within the finite elements undergoing localization. Multiple approaches have been proposed for finite elements with embedded discontinuities, see [22-24] for comparative studies. Among them is the assumed enhanced strain (AES) concept, first proposed in Simo and Rifai [25] and which has been successfully used to capture the mechanism of deformations in strain localization problems.

An appealing feature of the AES method is that no additional global degrees of freedom are required since the enhancements for discontinuities are condensed out locally. Two algorithms are readily available in the literature for condensation: the first one is based on the standard static condensation technique, where the discontinuities of the deformation mapping are condensed out at the element level, see for example [25,26,10,16,20,27-29]; an alternative way was recently proposed in [30-34], where the parameters defining the displacement jump within the finite element are condensed out at the material point level and the standard Galerkin approximation is utilized. In this paper we adopt a material point level condensation, where the resulting set of 
linearized constitutive equations are formally identical to those of standard continuum models [33]. This is a great advantage from an implementation point of view, since it only requires minor modifications to the material subroutine in an existing standard finite element code.

A major drawback afflicting all current post localization models, including AES, is the lack of proper material description within the band. Most implementations use simple constitutive formulations with constant material parameters, such as, the friction coefficient. Moreover, some form of softening law, requiring softening moduli selected arbitrarily and a priori, is generally required. A recent work by Foster et al. [35], which incorporates a variable friction coefficient into the AES formulation, makes a clear attempt to address this issue.

To overcome this drawback, we exploit the multiscale nature of granular materials. Multiscale approaches have recently surfaced in granular mechanics. Among others, a recent work by Chen et al. [36] investigates the mechanical behavior of granular materials within a multiscale framework using an internally-consistent probabilistic model. The work in [36] bridges scales at continuum level, but has not yet explicitly linked the continuum scale with the grain scale, which is known to be the fundamental scale for granular materials. To this end, the discrete element method (DEM), proposed by Cundall and Strack [37], was developed to capture the behavior of granular materials at the fundamental scale. The method has also been extensively used in modeling localization band in granular media, see for example [38-42], among others. However, as pointed out in [43], DEM suffers two major shortcomings: expensive computational cost and inability to capture real grain shapes. To overcome the shortcomings of DEM and combine the strengths of available continuum and discrete models, we resort to a recently proposed multiscale computational framework in [43-45] for modeling granular materials. The key idea is to bypass the phenomenological evolutions of material parameters in the continuum model, e.g. friction and dilatancy, and rather extract such evolutions directly from grain-scale structures and then upscale them into the continuum scale model. In this paper, we will reformulate the AES methodology within this multiscale computational framework, using a hierarchical information passing scheme. Following the taxonomy proposed by $\mathrm{Xu}$ [46], hierarchical means that the two scales are not coupled directly, but rather finer scales provide data that can be used in a sequential way as the calculations are coarsened or upscaled. By doing so, the evolutions of material parameters in the AES method could be provided based on the grain scale information. More importantly, phenomenological softening moduli are no longer required.

The remainder of the paper is structured as follows: in Section 2 , the kinematics induced by strong discontinuities and the constitutive equations of the AES method are briefly summarized; in Section 3, a hierarchical multiscale framework is first presented and the AES method is reformulated within the multiscale framework; in Section 4, two numerical examples are presented utilizing the multiscale framework to couple the AES with grain-scale computations and experiment data. Finally, we summarize our findings and make some concluding remarks in the conclusion section.

As for notations and symbols used in this paper, bold-faced letters denote tensors or vectors; the symbol ' $\because$ denotes an inner product of two vectors (e.g. $\boldsymbol{a} \cdot \boldsymbol{b}=a_{i} b_{i}$ ), or a single contraction of adjacent indices of two tensors (e.g. $\boldsymbol{c} \cdot \boldsymbol{d}=c_{i j} d_{j k}$ ); the symbol ' $\otimes$ ' denotes a juxtaposition (e.g. $\boldsymbol{a} \otimes \boldsymbol{b}=a_{i} b_{j}$, or $\boldsymbol{\alpha} \otimes \boldsymbol{\beta}=\alpha_{i j} \beta_{k l}$ ); the symbol ':' denotes an inner product of two second-order tensors (e.g. $\boldsymbol{c}$ : $\left.\boldsymbol{d}=c_{i j} d_{i j}\right)$; the symbol ' $\|\cdot\|$ ' denotes an $L_{2}$ norm of a vector, e.g. $\|\boldsymbol{e}\|=(\boldsymbol{e} \cdot \boldsymbol{e})^{1 / 2}$ or a tensor $\|\boldsymbol{A}\|=(\boldsymbol{A}: \boldsymbol{A})^{1 / 2}$. Stress and strain are expressed in Voigt notation, and as a result, the associated stiffness/compliance are expressed as matrices.

\section{Discontinuous kinematics and constitutive equations for the AES method}

In this section, discontinuous kinematics and constitutive equations for the AES method are briefly summarized. As mentioned in the introduction, material point level condensation will be utilized. For more detailed formulations and development of this method, including its variational and matrix formulation, we refer the interested reader to [30,31,33].

\subsection{Kinematics induced by strong discontinuities}

The domain of interest here is a body $\Omega$ split by a surface of discontinuity $\mathcal{S}$. As usual, $\Gamma_{u}$ and $\Gamma_{t}$ furnish the essential and natural boundaries and $\boldsymbol{n}$ is the unit normal of the discontinuity surface, pointing towards $\Omega_{+}$. An additional subdomain $\Omega^{h} \subset \Omega$ is defined by two arbitrary boundaries ahead $\left(\mathcal{S}_{+}^{h}\right)$ and behind $\left(\mathcal{S}_{-}^{h}\right)$ the discontinuity surface, and split by $\mathcal{S}$ into the subdomains $\Omega_{+}^{h}$ and $\Omega_{-}^{h}$. This subdomain defines the support of the ramp function in Eq. (2.1).

The discontinuous displacement field can be decomposed into a continuous part and a discontinuous part as

$\boldsymbol{u}(\boldsymbol{x})=\underbrace{\overline{\boldsymbol{u}}(\boldsymbol{x})}_{\text {continuous }}+\underbrace{M_{\mathcal{S}}(\boldsymbol{x}) \llbracket \boldsymbol{u} \rrbracket(\boldsymbol{x})}_{\text {discontinuous }}$,

where $\llbracket \boldsymbol{u} \rrbracket(\boldsymbol{x})$ is the displacement jump. The scalar function $M_{\mathcal{S}}(\boldsymbol{x})$ generates discontinuity on the surface $\mathcal{S}$ and is given by

$M_{\mathcal{S}}(\boldsymbol{x})=H_{\mathcal{S}}(\boldsymbol{x})-f^{h}(\boldsymbol{x}), \quad$ with $\operatorname{supp}\left[M_{\mathcal{S}}\right]=\Omega_{+}^{h} \cup \Omega_{-}^{h}$,

where "supp" represents the support of a function. The Heaviside function $H_{\mathcal{S}}(\boldsymbol{x})$ is defined on $\mathcal{S}$ such that

$H_{\mathcal{S}}(\boldsymbol{x})= \begin{cases}1 & \boldsymbol{x} \in \Omega_{+}, \\ 0 & \boldsymbol{x} \in \Omega_{-} .\end{cases}$

At the same rate, $f^{h}(\boldsymbol{x})$ is any arbitrary smooth function that satisfies the requirements

$f^{h}(\boldsymbol{x})= \begin{cases}1 & \boldsymbol{x} \in \Omega_{+} \backslash \Omega_{+}^{h}, \\ 0 & \boldsymbol{x} \in \Omega_{-} \backslash \Omega_{-}^{h} .\end{cases}$

A one-dimensional representation of the decomposed displacement field is shown in Fig. 1(b). Using such kinematic description of the displacement field, essential boundary conditions can be applied exclusively on $\overline{\boldsymbol{u}}(\boldsymbol{x})$. Therefore, it allows for the computed global nodal displacement $\overline{\boldsymbol{u}}(\boldsymbol{x})$ to be accepted as the final displacement field. The discontinuous part of the displacement field $\llbracket \boldsymbol{u} \rrbracket$ is condensed

(a)

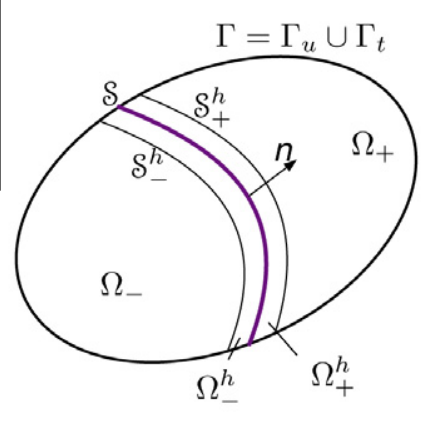

Fig. 1. (a) Domain $\Omega$ with a surface of discontinuity $\mathcal{S}$; (b) One-dimensional representation of the decomposed displacement field. (b)

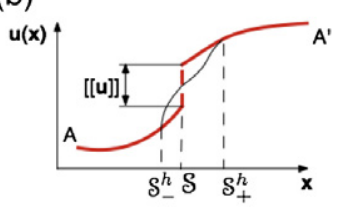

$\overline{\mathbf{u}}(\mathbf{x})$

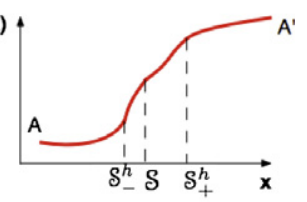

$\operatorname{Ms}[[u]](\mathbf{x}) \uparrow$

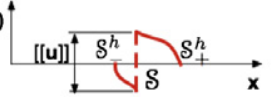


out at the material point level, leaving the global degrees of freedom unchanged. In the AES concept, the enriched displacement field is modeled in an incompatible sense. It is acceptable to neglect the gradient of the displacement discontinuity, i.e., $\nabla^{\mathrm{s}} \llbracket \boldsymbol{u} \rrbracket=\mathbf{0}$. Then, for infinitesimal deformations, the total strain rate tensor is written as

$\dot{\boldsymbol{\epsilon}}=\nabla^{\mathrm{s}} \dot{\boldsymbol{u}}=\nabla^{\mathrm{s}} \dot{\overline{\boldsymbol{u}}}-\left(\llbracket \dot{\boldsymbol{u}} \rrbracket \otimes \nabla f^{h}\right)^{\mathrm{s}}+\delta_{\mathcal{S}}(\llbracket \dot{\boldsymbol{u}} \rrbracket \otimes \boldsymbol{n})^{\mathrm{s}}$,

where $\delta_{\mathcal{S}}$ is the Dirac delta function on the surface $\mathcal{S}$. It should be pointed out that it is a modeling assumption to neglect the gradient of the displacement jump, and that, in general, it is possible to allow variations in the displacement jump within one element, as for example in $[47,48]$, where a linear interpolation for the displacement jump is used.

\subsection{Constitutive equations for post-localization response}

In this section, we will focus on the constitutive equations for post-localization response, since there is no difference between the constitutive framework used in the classical finite element method and the AES method at the pre-localization stage.

Once a material is localized, a post-localization model describing material response at the damaged state is invoked. In developing the constitutive equations, it is assumed that inelastic deformations induced by plasticity or material damage are restricted to the deformation band in question. A convex elastic domain $\widetilde{E}$ is defined by a smooth yield function $F$, characterizing the yield condition on the band,

$\widetilde{E}=\left\{(\boldsymbol{\sigma}, \boldsymbol{\alpha}) \in \mathbb{R}^{\text {ndim }} \mid F(\boldsymbol{\sigma}, \boldsymbol{\alpha}) \leqslant 0\right\}$,

where $\alpha$ is a stress-like vector of internal variables of dimension $n$. The yield function $F$ can be different from its counterpart at the intact stage. In addition, a plastic potential function $G$ can be defined. The model does not require an explicit form for this function, but some restrictions apply on $G$, as will be shown in Eq. (2.10).

Decomposing the displacement jump rate $\llbracket \dot{\boldsymbol{u}} \rrbracket$ into its magnitude $\dot{\xi}$ and direction vector $\boldsymbol{m}$ we obtain

$\llbracket \dot{\boldsymbol{u}} \rrbracket=\dot{\xi} \boldsymbol{m}$,

from which expression the associated strain rate tensor emanates, cf., Eq. (2.1)

$\dot{\boldsymbol{\epsilon}}=\nabla^{\mathrm{s}} \dot{\boldsymbol{u}}=\nabla^{\mathrm{s}} \dot{\overline{\boldsymbol{u}}}-\dot{\xi}\left(\boldsymbol{m} \otimes \nabla f^{h}\right)^{\mathrm{s}}+\dot{\xi} \delta_{\mathcal{S}}(\boldsymbol{m} \otimes \boldsymbol{n})^{\mathrm{s}}$.

Then, by subtracting the plastic part, we obtain the elastic strain rate tensor $\dot{\boldsymbol{\epsilon}}^{e}$

$\dot{\boldsymbol{\epsilon}}^{e}=\nabla^{\mathrm{s}} \dot{\overline{\boldsymbol{u}}}-\dot{\xi}\left(\boldsymbol{m} \otimes \nabla f^{h}\right)^{s}+\dot{\xi} \delta_{\mathcal{S}}(\boldsymbol{m} \otimes \boldsymbol{n})^{s}-\lambda_{\delta} \delta_{\mathcal{S}} \frac{\partial G}{\partial \boldsymbol{\sigma}}$,

where $\lambda=\lambda_{\delta} \delta_{\mathcal{S}}$ is the usual plastic consistency parameter.

The last two terms of (2.9) cancel because of the assumption that plasticity is localized to the discontinuity and hence the slip rate tensor $\dot{\xi}(\boldsymbol{m} \otimes \boldsymbol{n})^{s}$ is fully plastic, which actually imposes a constraint on the plastic potential function $G$

$\frac{\partial G}{\partial \boldsymbol{\sigma}}=\Lambda(\boldsymbol{m} \otimes \boldsymbol{n})^{\mathrm{s}}, \quad \Lambda=\frac{\dot{\xi}}{\lambda_{\delta}}$.

The Cauchy stress rate tensor is then computed as

$\dot{\boldsymbol{\sigma}}=\boldsymbol{c}^{E}: \dot{\boldsymbol{\epsilon}}^{e}=\boldsymbol{c}^{E}:\left[\nabla^{S} \dot{\overline{\boldsymbol{u}}}-\dot{\xi}\left(\boldsymbol{m} \otimes \nabla f^{h}\right)^{s}\right], \quad$ in $\Omega \backslash \mathcal{S}$,

where $c^{E}$ is the elastic constitutive tensor. The constitutive equation resembles the predictor-corrector scheme of continuum plasticity, where $\dot{\xi}$ plays the role of the plastic consistency parameter. Yielding in the damaged state is described by the consistency condition on the band,
$\dot{F}=\boldsymbol{\psi}: \dot{\boldsymbol{\sigma}}-\dot{\xi} \widetilde{H}_{\delta}=0, \quad \boldsymbol{\psi}=\frac{\partial F}{\partial \boldsymbol{\sigma}}$,

where $\widetilde{H}_{\delta}$ is the softening modulus on the band. Substituting Eq. (2.11) into (2.12) and solving for the jump rate $\dot{\xi}$,

$$
\dot{\xi}=\frac{\psi: \boldsymbol{c}^{E}: \dot{\overline{\boldsymbol{\epsilon}}}}{\psi: \boldsymbol{c}^{E}:\left(\boldsymbol{m} \otimes \nabla f^{h}\right)^{s}+\widetilde{H}_{\delta}}, \quad \dot{\overline{\boldsymbol{\epsilon}}}=\nabla^{s} \dot{\overline{\boldsymbol{u}}} .
$$

Then, the Cauchy stress rate tensor, at the post-localization stage, is written as

$\dot{\boldsymbol{\sigma}}=\tilde{\boldsymbol{c}}^{E P}: \nabla^{S} \dot{\overline{\boldsymbol{u}}}$,

where $\tilde{\boldsymbol{c}}^{E P}$ is the equivalent elastoplastic tangential modulus with the presence of displacement jumps and is given as

$\tilde{\boldsymbol{c}}^{E P}=\boldsymbol{c}^{E}-\frac{\boldsymbol{c}^{E}:\left(\boldsymbol{m} \otimes \nabla f^{h}\right)^{S} \otimes \boldsymbol{\psi}: \boldsymbol{c}^{E}}{\boldsymbol{\psi}: \boldsymbol{c}^{E}:\left(\boldsymbol{m} \otimes \nabla f^{h}\right)^{S}+\widetilde{H}_{\delta}}$.

The constitutive framework implied by the yield function $F$, plastic flow (Eq. (2.10)) and the softening modulus $H_{\delta}$ plays a crucial role in the accuracy of the AES method. Currently, constant material properties are usually assumed a priori and selection of values is quite arbitrary. This is one of the major drawbacks of the AES method. To overcome this, we will resort to the multiscale nature of granular materials and linking the underlying grain-scale information with the continuum scale AES method.

\section{AES for multiscale framework}

In this section, the standard AES method will be reformulated within a recently-proposed multiscale framework for granular materials, see for example [43-45]. The most salient difference between the algorithm presented herein and that in the standard AES formulation is that the softening modulus $H_{\delta}$ is no longer required in the current framework. Moreover, the evolutions of the plastic internal variables will be extracted from the grain-scale information, instead of assuming their values a priori.

\subsection{Hierarchical multiscale framework}

In this section, a hierarchical multiscale framework is presented to couple continuum-scale plasticity models with information extracted from grain-scale kinematics, e.g. DEM computations or physical experiments.

Continuum description considers the classical two-invariant Drucker-Prager (D-P) model, where the two stress invariants are given as

$p=\frac{1}{3} \operatorname{tr} \boldsymbol{\sigma} ; \quad q=\sqrt{\frac{3}{2}}\|\boldsymbol{s}\|$

with $\operatorname{tr} \square=\square: \delta$ as the trace operator, $\boldsymbol{s}=\boldsymbol{\sigma}-p \boldsymbol{\delta}$ as the deviatoric component of the stress tensor and $\|\square\|$ denotes the $L_{2}$ norm of $\square$. Similarly, the invariants of the strain rate tensor are defined as

$\dot{\epsilon}_{v}=\operatorname{tr} \dot{\boldsymbol{\epsilon}} ; \quad \dot{\epsilon}_{s}=\sqrt{\frac{2}{3}}\|\dot{\boldsymbol{e}}\|$,

where $\dot{\boldsymbol{e}}=\dot{\boldsymbol{\epsilon}}-1 / 3 \dot{\epsilon}_{v} \boldsymbol{\delta}$ is the deviatoric component of the strain rate tensor.

Using the aforementioned invariants of the stress tensor, the yield function $F$ and the plastic potential function $G$ for the D-P model at the pre-localization stage can be defined as

$F(\boldsymbol{\sigma}, \mu)=q+\mu p-c_{f}=0$,

$G(\boldsymbol{\sigma}, \beta)=q+\beta p-c_{q}$ 


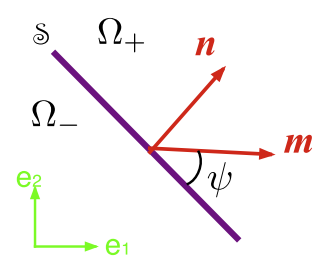

Fig. 2. Relation between $\boldsymbol{m}$ and $\boldsymbol{n}$ relative to the discontinuity surface $\mathcal{S}$.

where the cohesion parameter $c_{f}=0$ for granular materials and the cohesion-like parameter $c_{q}$ ensures that the potential surface $G$ is always attached to the current stress point. Two plastic internal variables are involved in the D-P model: the friction coefficient $\mu$ and the dilatancy parameter $\beta$. Physically, $\mu$ directly represents the mobilized friction angle of granular materials. At yielding, $\mu$ takes the form

$\mu=-\frac{q}{p}$.

The dilatancy parameter $\beta$ measures the change in volumetric plastic deformation for a given change in deviatoric plastic deformation. Specifically,

$\beta=\frac{\dot{\epsilon}_{v}^{p}}{\dot{\epsilon}_{s}^{p}} \approx \frac{\dot{\epsilon}_{v}}{\dot{\epsilon}_{s}}$,

where in approximation, the elastic strain increments are neglected. This is a plausible approximation once plasticity dominates the deformation, which is the case for most granular materials after yielding. It is important to clarify that Eq. (3.6) is only used to extract $\beta$ from granular scale computations or observations. This assumption only affects the accuracy of extracted value from granular scale. In the continuum scale computation, we do not enforce this assumption. As can be seen from the examples presented herein, this method yields good accuracy relative to experiments and direct observations.

In our case, post-localization, the same form of the yield function as in Eq. (3.3) is used, but different yield functions can be chosen, if desired. As discussed in Section 2.2, a specific form of the plastic potential function is not required. Instead, the direction of the plastic flow is provided by

$\frac{\partial G}{\partial \boldsymbol{\sigma}} \propto(\boldsymbol{m} \otimes \boldsymbol{n})^{s}$,

where the relation between $\boldsymbol{m}$ and $\boldsymbol{n}$ is shown in Fig. 2. Furthermore, the jump's dilatancy angle $\psi$ (see $[29,28]$ for a closer definition) controls the distance between $\boldsymbol{m}$ and the slip surface $\mathcal{S}$. The specific geometrical relation between $\boldsymbol{m}$ and $\boldsymbol{n}$ is given by

$\boldsymbol{m} \cdot \boldsymbol{n}=\sin \psi$.

Remark 1. The procedure to evaluate $m$ and $n$, which are central to the AES formulation, deserves further explanation. In the numerical examples presented in this paper, the orientation of the shear band (e.g. given by $n$ ) is readily available from DEM computations or experimental data by measuring the angle of the formed localization band. In this sense, the DEM is seen as a numerical experiment. Therefore, there are similarities between extracting shear band orientation and local information from DEM and from physical experiment. Also, for both physical experiment and the DEM simulation, we know exactly when the localization is triggered and the multiscale computation will read localized data once the localization is reached. Alternatively, a more sophisticated pre-localization analysis of the acoustic tensor can be performed, signaling the onset of strain localization and providing the orientation of the band, see for example $[28,29,49]$. This latter analysis would be particularly useful when shear band information is not available or to make computations truly predictive. Here, we focus on post-bifurcation response and assume the orientation of the bands are known.

In standard AES, material properties, such as the aforementioned plastic internal variables, are usually assumed to be constant or to evolve with some arbitrary phenomenological relation. Moreover, an additional softening modulus $H_{\delta}$ is typically required so that the softening behavior after localization is captured. Within the multiscale framework, however, we exploit the physical significance of the plastic internal variables to extract them directly from grain-scale information available form DEM calculations or data from physical experiments.

In the case of DEM computations, the friction coefficient and dilatancy parameter are calculated based on average micromechanical stress and strain tensors [50-52]. Following the procedure presented in [43], $\mu$ and $\beta$ are calculated as

$\mu \simeq-\frac{\bar{q}}{\bar{p}} ; \quad \beta \simeq \frac{\Delta \bar{\epsilon}_{v}}{\Delta \bar{\epsilon}_{s}}$,

where $\square$ signifies micro-mechanically-based quantities; $\Delta \square=\square_{n+1}$ $-\square_{n}$ represents the change of $\square$ from time $t_{n}$ to $t_{n+1}$.

In the case of experiment-based computations, only dilatancy can be estimated directly. In the example presented herein, we use the data from Mooney et al. [53], who measured the change in volumetric and deviatoric strains, and related it to the angle of dilatancy as (cf. Eq. (6) in [53]),

$\psi=\tan ^{-1}\left(\frac{\Delta \epsilon_{v}}{\Delta \epsilon_{s}}\right)$

and then $\beta$ is directly related to the dilation angle via

$\beta=\tan \psi$.

Remark 2. Recent advances in X-ray tomography and digital image correlation allow the kinematics of each grain to be captured throughout experiments and in real time, e.g. [54,55]. In this case, the incremental strain field can be calculated using finite element interpolations and the incremental displacement data is obtained directly from experiments. Then, $\beta$ is calculated from Eq. (3.9). This method is fully explored in a recent paper by Andrade et al. [45].

Regarding the friction coefficient, since the micro-mechanical stress tensor is not readily available from experiments, one indirect way to estimate $\mu$ is to invoke a stress-dilatancy relation for granular materials [56]

$\mu=\beta+\mu_{\mathrm{cv}}$,

where $\mu_{\mathrm{cv}}$ is a constant material parameter measuring the friction coefficient or stress ratio at critical state in a granular assembly.

Once evolutions of the plastic internal variables are fully defined, they will be used hierarchically as the calculations are coarsened or upscaled to the continuum scale. One of the key features of this multiscale framework is that the plastic internal variables are "frozen" within each time step, and only updated when global convergence is achieved. Fig. 3 shows the flow chart for the multiscale framework within a finite element program. Numerical implementation for pre-localization using conventional finite element method has been presented in recent publications by the authors $[43,44]$. For post-localization analyses using the AES method, we will extend the previously-developed algorithms and present them in detail in the next section.

In a nutshell, the hierarchical multiscale framework consists of the following key steps: (1) perform grain-scale computation using DEM, or obtain local measurement data from experiment; (2) extract evolutions of plastic internal variables from DEM using Eq. (3.9), or from experiments using either displacement field or measured dilatancy angle to obtain $\beta$, and use Eq. (3.12) to obtain 


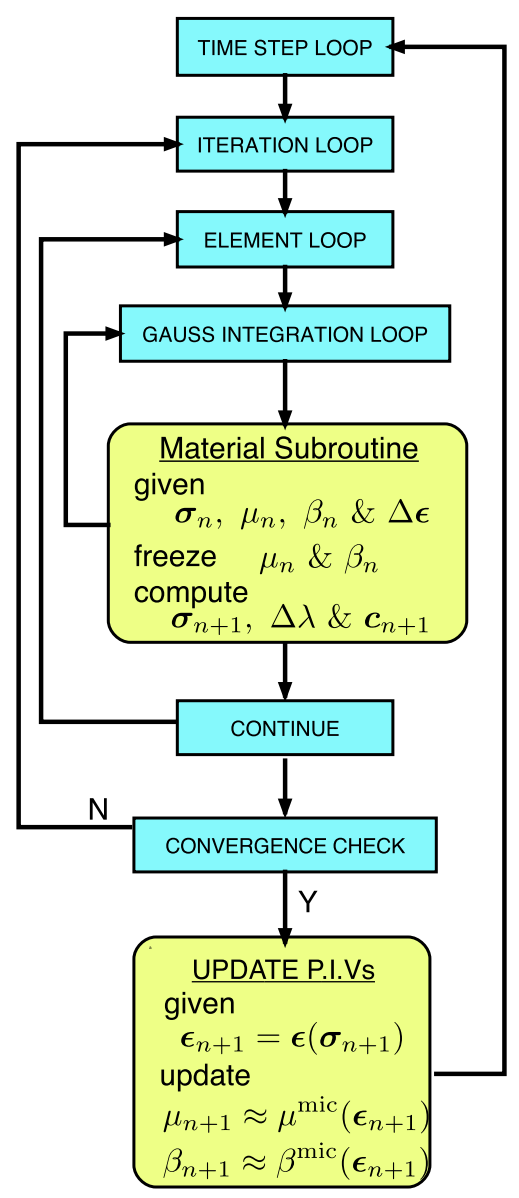

Fig. 3. Flowchart for the hierarchical multiscale scheme, modified from [44].

$\mu$; (3) use these evolutions of plastic internal variables as input for continuum scale computation using FEM, with localized elements enhanced through the AES technique.

Remark 3. One advantage of the aforementioned framework is that it provides insight into the granular scale information and a way to link it to continuum scale AES, wherever and whenever is necessary. This has not yet been taken into account by the standard AES. Also, a hierarchical multiscale framework has been presented in this section, but the framework could be extended to concurrent information passing schemes, as shown in [44]. This would allow AES to be linked with granular computation on the fly to achieve truly predictive capability.

\subsection{Stress integration algorithm for AES within the multiscale framework}

As shown in Fig. 3, within each time step, the plastic internal variables are "frozen", and are only updated at global convergence. Therefore, the integration algorithm at each material point is analogous to that of a perfect plasticity model. Because of the delay in updating the plastic internal variables, the consistency condition (Eq. (2.12)) is no longer enforced when integrating stresses. Instead, the yielding condition $F_{n+1}=0$ will be enforced.

To compute the stress state at time $t_{n+1}$, we will start with the rate form of the Cauchy stress. In AES formulation, the Cauchy stress rate is given as (cf. Eq. (2.11))

$\dot{\boldsymbol{\sigma}}=\dot{\boldsymbol{\sigma}}^{\mathrm{tr}}-\dot{\xi} \boldsymbol{c}^{E}:\left(\boldsymbol{m} \otimes \nabla f^{h}\right)^{S}, \quad \dot{\boldsymbol{\sigma}}^{\mathrm{tr}}=\boldsymbol{c}^{E}: \nabla^{S} \dot{\overline{\boldsymbol{u}}}$, where $\dot{\boldsymbol{\sigma}}^{\mathrm{tr}}$ is the trial stress rate. Integrating Eq. (3.13) from $t_{n}$ to $t_{n+1}$, we obtain

$\boldsymbol{\sigma}_{n+1}=\boldsymbol{\sigma}_{n+1}^{\mathrm{tr}}-\Delta \xi \boldsymbol{c}^{E}:\left(\boldsymbol{m} \otimes \nabla f^{h}\right)^{s}$,

where $\boldsymbol{\sigma}^{\mathrm{tr}}{ }_{n+1}$ is the trial stress at time $t_{n+1}$ and is written as

$\boldsymbol{\sigma}_{n+1}^{\mathrm{tr}}=\boldsymbol{\sigma}_{n}+\boldsymbol{c}^{E}:\left(\nabla \overline{\boldsymbol{u}}_{n+1}^{h}-\nabla \overline{\boldsymbol{u}}_{n}^{h}\right)^{s}$.

As mentioned before, yielding condition on the band is enforced instead of the consistency condition, such that,

$F_{n+1}=F\left(\boldsymbol{\sigma}_{n+1}, \mu_{n}\right)=0$.

Note that the plastic variable $\mu$ is held at its value at the previous time step $t_{n}$, resulting in a delayed update. The integration algorithm is based on finding the stress state $\boldsymbol{\sigma}_{n+1}$ so that Eqs. (3.14) and (3.16) are satisfied. The algorithm is summarized in the following boxes.

Box 1. Stress integration algorithm for the AES method within the multiscale framework

GIVEN: $\boldsymbol{\sigma}_{n}, \nabla \overline{\boldsymbol{u}}_{n+1}^{h}, \nabla \overline{\boldsymbol{u}}_{n}^{h}, \nabla f^{h}, \boldsymbol{m}$ and $\mu_{n}$

FIND: $\sigma_{n+1}$ and $\Delta \xi$ such that Eqs. (3.14) and (3.16) are satisfied

STEP 1. Compute the trial stress state $\boldsymbol{\sigma}_{n+1}^{\mathrm{tr}}$, and the yield function $F^{\operatorname{tr}}\left(\boldsymbol{\sigma}_{n+1}^{\mathrm{tr}}, \mu_{n}\right)$

STEP 2. Check the yielding condition: $F^{\operatorname{tr}}\left(\boldsymbol{\sigma}_{n+1}^{\mathrm{tr}}, \mu_{n}\right) \leqslant 0$ ? Yes, set $\boldsymbol{\sigma}_{n+1}=\boldsymbol{\sigma}_{n+1}^{\mathrm{tr}}$ and exit

STEP 3. No, initialize $\Delta \xi=0$ and use Newton-Raphson to solve for $\Delta \xi$ (see Box 2)

STEP 4. Update $\boldsymbol{\sigma}_{n+1}=\boldsymbol{\sigma}_{n+1}^{\mathrm{tr}}-\Delta \xi \boldsymbol{c}^{E}:\left(\boldsymbol{m} \otimes \nabla f^{h}\right)^{S}$

STEP 5. Compute the consistent tangent operator (CTO): $\boldsymbol{c}_{n+1}=\frac{\partial \boldsymbol{\sigma}_{n+1}}{\partial \nabla^{s} \overline{\boldsymbol{u}}_{n+1}^{h}}$ (see details below)

Box 2. Newton-Raphson loop to solve for $\Delta \xi$

STEP 1. Initialize the unknown $\Delta \xi^{k}=0$ and set the iteration number $k=0$

STEP 2. Compute $\boldsymbol{\sigma}_{n+1}^{k}=\boldsymbol{\sigma}_{n+1}^{\mathrm{tr}}-\Delta \xi^{k} \boldsymbol{c}^{E}:\left(\boldsymbol{m} \otimes \nabla f^{h}\right)^{s}$ and $F_{n+1}^{k}\left(\boldsymbol{\sigma}_{n+1}^{k}, \mu_{n}\right)$

STEP 3. Check if the residual $r\left(\boldsymbol{\sigma}_{n+1}^{k}, \Delta \xi^{k}\right)=$ $F_{n+1}^{k}\left(\boldsymbol{\sigma}_{n+1}^{k}, \mu_{n}\right)=0$ ? Yes, set $\Delta \xi=\Delta \xi^{k}$ and exit

STEP 4. No, compute the local consistent tangent $r^{\prime}=-\boldsymbol{b}$ : $\boldsymbol{a}$ where $\boldsymbol{a}=-\frac{\partial \boldsymbol{\sigma}_{n+1}}{\partial \Delta \boldsymbol{\xi}}$ and $\boldsymbol{b}=\frac{\partial F_{n+1}}{\partial \boldsymbol{\sigma}_{n+1}}$.

STEP 5. Update $\Delta \xi^{k+1}=-\left(r^{\prime}\right)^{-1} r+\Delta \xi^{k}, k=k+1$ and go to STEP 2

In Box 1, the consistent tangent operator (CTO) $\boldsymbol{c}_{n+1}$ is needed in order to achieve optimal asymptotically quadratic convergences rate at the global level. For the stress update presented in Eq. (3.14), the CTO can be computed as

$\boldsymbol{c}_{n+1}=\frac{\partial \boldsymbol{\sigma}_{n+1}}{\partial \nabla^{S} \overline{\boldsymbol{u}}_{n+1}^{h}}=\boldsymbol{c}^{E}-\frac{\boldsymbol{a} \otimes \tilde{\boldsymbol{a}}}{\boldsymbol{b}: \boldsymbol{a}}$,

where

$\boldsymbol{a}=-\frac{\partial \boldsymbol{\sigma}_{n+1}}{\partial \Delta \xi}, \quad \boldsymbol{b}=\frac{\partial F_{n+1}}{\partial \boldsymbol{\sigma}_{n+1}}, \quad \tilde{\boldsymbol{a}}=\boldsymbol{b}: \boldsymbol{c}^{E}$. 


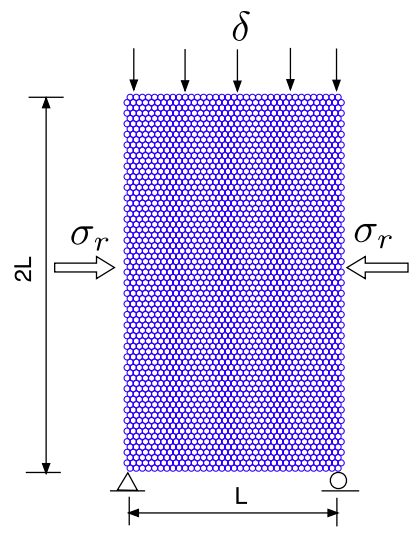

Fig. 4. Initial configuration and boundary conditions under plane strain loading of the DEM model.

\section{Numerical examples}

In this section, we present two numerical examples to illustrate the applicability of the reformulated AES method within the hierarchical multiscale framework and to analyze its numerical performance. Both examples are plane strain compression tests. The first one utilizes DEM as the micromechanical model to extract evolutions of plastic internal variables, while the second one obtains such information directly from a well-instrumented physical experiment. In each example, different meshes are generated to demonstrate the objectivity of this framework with respect to mesh refinement and insensitivity to mesh alignment.

\subsection{Coupling AES with DEM}

In this example, a plane strain compression test coupling AES with DEM is presented. DEM is used as the microscale model to provide evolutions of the plastic internal variables. We chose two-dimensional (2D) DEM for its computational simplicity and efficiency. To this end, the stress and strain invariants given in Eqs. (3.1) and (3.2) will be redefined for 2D cases.

The 2D DEM model consists of 2520 disks, with the same radius of $0.05 \mathrm{~m}$. The dimensions of the sample are $0.31 \mathrm{~m}$ by $0.62 \mathrm{~m}$. The sample is initially consolidated under stress controlled boundary conditions until $\sigma_{a}=\sigma_{r}=-100 \mathrm{kPa}$, where $\sigma_{a}$ and $\sigma_{r}$ are the axial and lateral stresses. After consolidation stage, $\sigma_{r}$ is held constant while the top platen moves vertically downward under prescribed incremental strain, with the bottom plate fixed. The loading continues after a localization band appears and stops when critical state is apparently reached. The initial configuration and boundary conditions under plane strain loading for the DEM model are shown in Fig. 4.

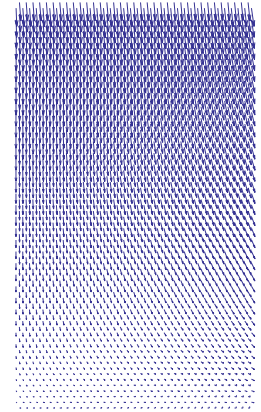

$\epsilon_{a}=0.20 \%$
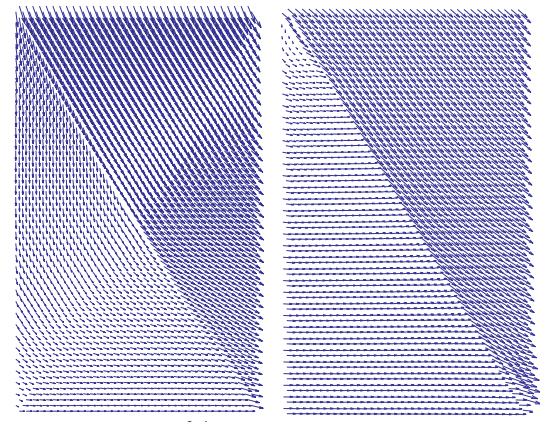

$\epsilon_{a}=0.50 \%$

Fig. 6. Relative displacement fields at different axial strain levels for the DEM simulation.

For 2D, we use the following definition for stress invariants, see also [57] for similar definitions for 2D biaxial test:

$p=\sigma_{r}+\sigma_{a} ; \quad q=\sigma_{r}-\sigma_{a}$.

Similarly, for strain invariants

$\epsilon_{v}=\epsilon_{\mathrm{r}}+\epsilon_{a} ; \quad \epsilon_{\mathrm{s}}=\epsilon_{\mathrm{r}}-\epsilon_{a}$.

Using the 2D definitions of $p$ and $q$ in the D-P yield surface in Eq. (3.5) the friction coefficient takes the form

$\mu=\frac{\sigma_{r}-\sigma_{a}}{\sigma_{r}+\sigma_{a}}=\sin \phi$

where $\phi$ is called the friction angle, and it sets the angle of the failure envelope under Mohr-Coulomb failure criterion.

Fig. 5 shows the global stress-strain behavior for this DEM simulation. The axial stress increases linearly with the axial strain up to $0.5 \%$, where the stress reaches its peak value. Marked softening follows the peak. As will be seen later in the displacement field (see Fig. 6), the initially homogeneous deformation breaks down at the peak stress, after which point the localization band starts to form. This band weakens the whole sample and contributes to the softening behavior seen in the axial stress. We will assume the sample undergoes elastic deformation up to $0.5 \%$ axial strain. Fig. 5(b) shows that the volumetric strain decreases first (contracting behavior) and then increases (dilating behavior) until it approaches a plateau (critical state), as typically seen in a plane strain compression test in relatively dense granular materials. The particular shapes of stress strain response and the fact that peak stress not corresponding to the inflexion point of volumetric strain curve, are the results of the simplified DEM model we have chosen, i.e., equal radius disks arranged in a highly structured pattern. If a more sophisticated DEM is used, the behavior would be closer to that of real granular materials.

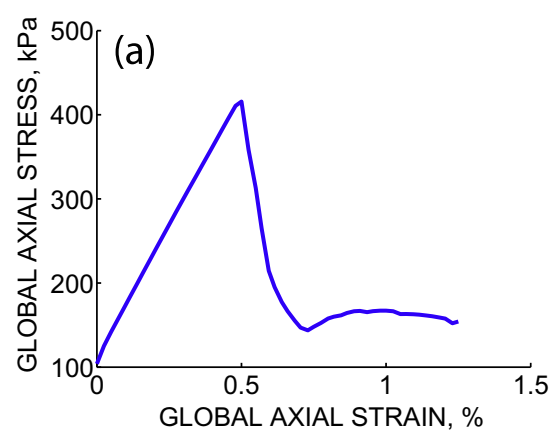

Fig. 5. Stress-strain behavior of the DEM simulation in plane strain compression test: (a) Axial stress vs. axial strain; (b) Volumetric strain vs. axial strain. 


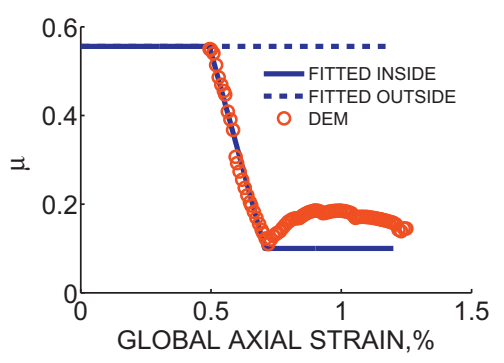

(a) Evolution off riction from DEM

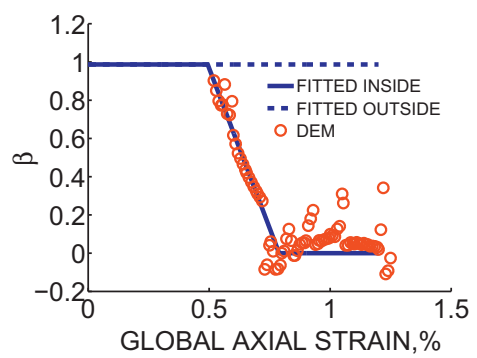

(b) Evolution of dilatancy from DEM

Fig. 7. Evolutions of plastic internal variables from DEM computation.

To pictorially show the mechanism of localization band formation in the DEM simulation, relative displacements at the center of the DEM disks are plotted at various stages of deformation in Fig. 6. Here, relative displacements are computed as the difference between the current position particles and their values at the previous time step. In the figure, $\epsilon_{a}$ stands for axial strain. As seen in Fig. 6, the sample deforms homogeneously up to the peak stress, where the axial strain level is about $0.5 \%$. Then, a single localization band starts to form and breaks the homogeneous deformation. This single band persists throughout the simulation.

It is enlightening to compare the angle of the shear band obtained from the DEM simulation with those from analytical expressions. The DEM simulation results in a band of approximately $63^{\circ}$ from the horizontal direction. If we adopt Mohr-Coulomb failure hypothesis, the failure plane would form an angle $\alpha_{f}$ from the plane of the major principal stress

$\alpha_{f}=45+\frac{\phi}{2}$.

From Eq. (4.3), $\sin \phi=\mu \approx 0.56$, which gives $\phi \approx 34^{\circ}$. Therefore, $\alpha_{f} \approx 62^{\circ}$. Also, if we compute $\boldsymbol{n}$ based on localization analysis [6], we will obtain an angle of shear band of approximately $60^{\circ}$, which is fairly close to the DEM result.

As shown in Section 3.1, friction and dilatancy can be calculated from DEM computation using Eq. (3.9), with definitions for stress and strain invariants in Eqs. (4.1) and (4.2), and shown in Fig. 7. Note that constant values are assumed for materials outside the band. This assumption will be immaterial because once localization happens, all materials outside of the localization band will unload elastically. Linear fits for both friction and dilatancy are used here for simplicity, but higher order interpolation functions could be incorporated easily.

The FE model is set up with the same sample dimension and boundary conditions. The material is described by a Drucker-Prager-type model, with evolutions of friction and dilatancy from Fig. 7. Other material parameters used are two elastic constants: Young's modulus $E=60 \mathrm{MPa}$ and Poisson's ratio $v=0.3$, both computed based on the elastic portion of the stress-strain behavior from DEM simulations.

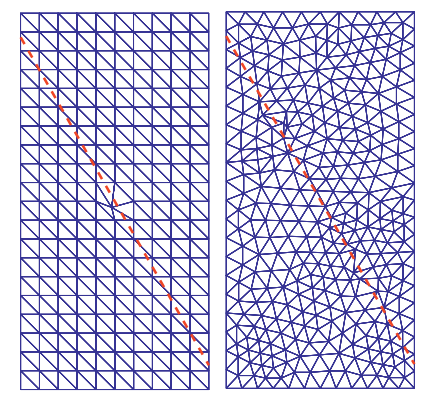

Fig. 8. Meshes used in plane strain simulations coupled with DEM.

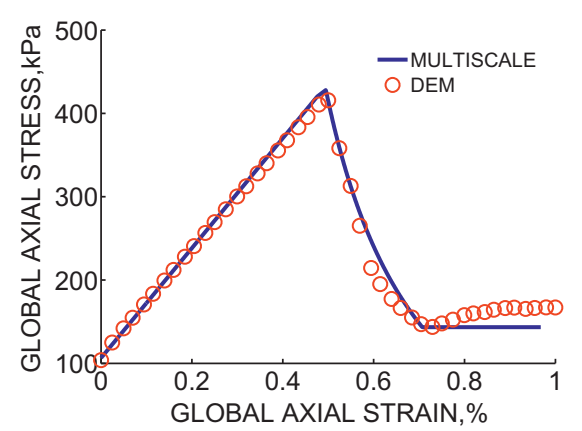

Fig. 9. Comparison of global stress-strain behavior: multiscale vs. DEM.

In the multiscale simulations, all elements are provided with the same evolution of friction and dilatancy up to $0.5 \%$ global axial strain. Once localization is reached at $0.5 \%$ axial strain, elements crossed by the localization band are equipped with enrichment furnished by the AES method. The evolution of the friction and dilatancy for those enhanced elements will be given by the solid line in Fig. 7. Dashed line is used for regular elements outside the band.

To illustrate the mesh-insensitive nature of the method, two different meshes are used as shown in Fig. 8. The dashed line is the potential localization band, as observed from DEM simulation. Fig. 9 shows the comparison of the global stress-strain behavior for multiscale simulations and DEM results. Note that DEM is used as the benchmark for verifying the performance of this multiscale framework. It can be seen that the multiscale model does a great job both pre- and post- localization. It captures the peak stress and the softening behavior very well. Also, it should be pointed out that two different finite element meshes produce identical results.

Remark 4. It should be pointed out that the discontinuity line observed in the DEM computation propagates across two offdiagonal corners. In the finite element mesh, the discontinuity is intentionally placed to avoid the corners. This is because, in the AES method, discontinuities propagating to essential boundaries will have difficulty converging. We did this by rotating the discontinuity line counter-clockwise so that there is at least one element between the discontinuity line and the essential boundary. In the current meshes shown, the discontinuity line is rotated about $3^{\circ}$. This angle could be reduced if finer meshes are used.

Deviatoric strain contours at $1 \%$ global axial strain are plotted for both meshes in Fig. 10. As expected, the deviatoric strains are concentrated in the localization band. The sample essentially behaves like two rigid blocks sliding with respect to each other, which mimics what happens in the direct numerical simulation using DEM. 

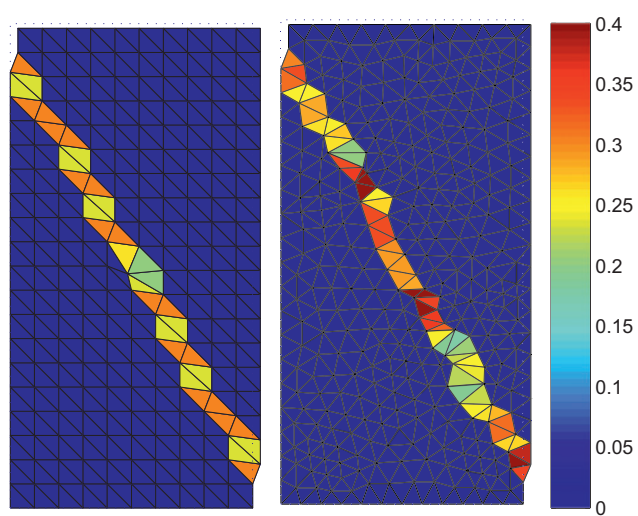

Fig. 10. Deviatoric strain contour at $1 \%$ global axial strain.

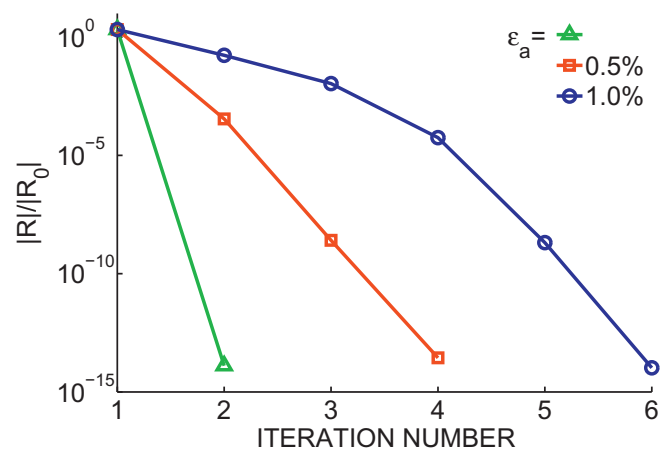

Fig. 11. Global convergence profile at three different axial strain level.

Finally, the global convergence profiles at three different axial strain levels are plotted in Fig. 11, where $R$ is the global residual and $R_{0}$ is the value of $R$ at the very first iteration. It can be seen that the desired asymptotic quadratic convergence rate is achieved, demonstrating the efficiency of the proposed framework.

\subsection{Coupling AES with experiments}

The previous example illustrates the coupling of AES with DEM within the multiscale framework. As will be seen in the second example, one of the most promising features of this multiscale framework is that it allows data from experimental measurements to be incorporated directly.

In this section, the behavior of a physical experiment of dense sand under drained plane strain compression is analyzed. The experiment was performed by Mooney et al. [53] using a wellinstrumented device. The sample dimensions are $140 \times 40 \times$ $80 \mathrm{~mm}$. Plane strain is enforced by two rigid walls, in the $80 \mathrm{~mm}$ direction. The sample was initially consolidated anisotropically with axial stress $\sigma_{a}=-210 \mathrm{kPa}$, and lateral stress $\sigma_{r}=-105 \mathrm{kPa}$. After consolidation, lateral stress is kept constant while the top plate moves down under displacement control. A localization band inclined at $63^{\circ}$ from the horizontal axis was observed when the global axial strain reached about $3 \%$. Dilation angle $\psi$ within the band was extracted using stereophotogrammetry, shown as red circles in Fig. 12.

In the numerical simulations, the material behavior is described by a Drucker-Prager model, same as in the first example. As discussed in Section 3.1, the dilatancy parameter $\beta$ is related to the angle of dilatancy $\psi$ by Eq. (3.11). Since there is no local measurement of either forces or stresses, the friction parameter $\mu$ can not be obtained directly from the experimental measurements.

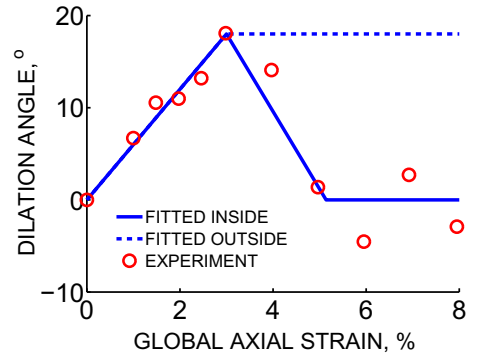

Fig. 12. Evolution of dilation angle observed in the experiment. (a)

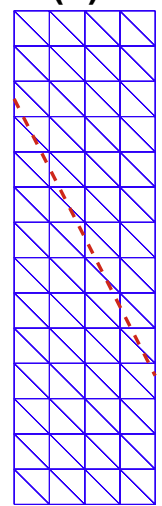

(b)

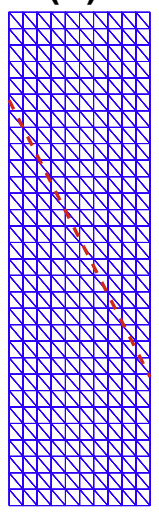

(c)

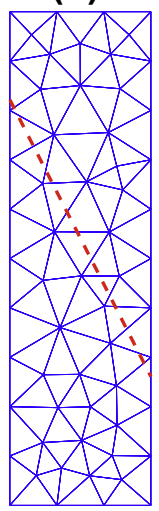

(d)

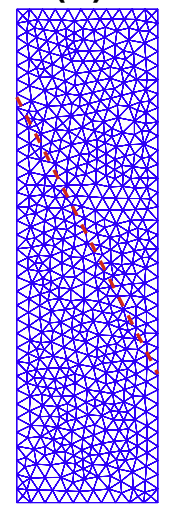

Fig. 13. Meshes used in plane strain compression test coupled with experimental data.

Instead, the stress-dilatancy relationship in (3.12) is used. In this study, $\mu_{\mathrm{cv}}=1.15$ is obtained from the experimental results.

In sum, material parameters input in the model are $E=40,000 \mathrm{kPa}, v=0.2, \mu_{\mathrm{cv}}=1.15$, and $\beta$ from Fig. 12 . Also, the orientation and location of the potential localization band are specified in the model based on experiment observations for the sake of simplicity.

Similar to the first example, all elements are provided with the same evolution of dilation angle up to $3 \%$ global axial strain. Once localization is reached, elements crossed by the localization band will be equipped with enrichment using the AES concept. The evolution of the dilation angle for those enhanced elements will be given by the solid line in Fig. 12. The dashed line on the figure represents elements outside the localization band. Since the sample unloads elastically outside the band, the assumption of a constant dilation angle will not affect the results. It should be

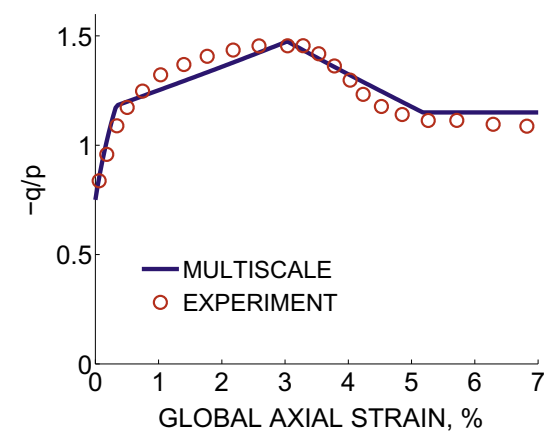

Fig. 14. Evolution of stress ratio with global axial strain. 


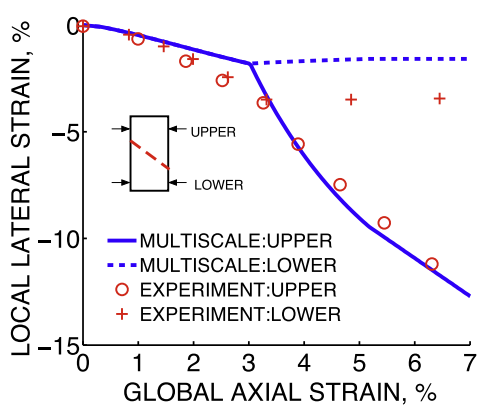

Fig. 15. Lateral strain vs. axial strain. (a)

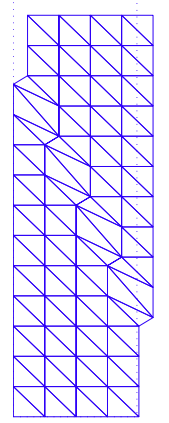

(b)

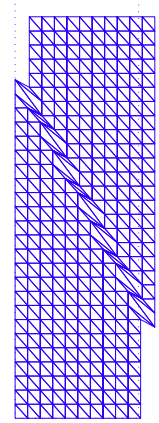

(c)

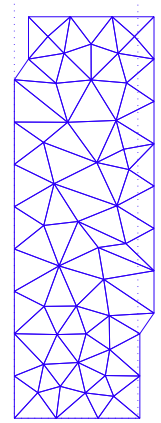

(d)

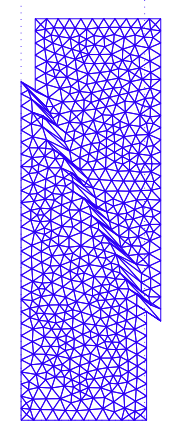

Fig. 16. Deformed samples for plane strain simulations.

pointed out the standard AES method would capture the behavior by fitting parameters. However, as mentioned in Remark 3, the multiscale provides insight and a way to link the granular scale information for true predictiveness without phenomenology.

To illustrate the mesh-insensitive feature of the method, four different meshes are used as shown in Fig. 13. The dashed line is the potential localization band, as observed in the experiment. Fig. 14 shows the evolution of the stress ratio $-q / p$ for both simulation and experiment, where the values from the simulation correspond to the global averaged stresses. It is clear that the multiscale simulation captures the global stress response remarkably well. Also, four different meshes produce identical results. It should be clarified that in the example, the discontinuity orientation and placement are selected a priori and made to be the same for each mesh. When a propagation criterion is implemented, the results from different meshes may not be identical.

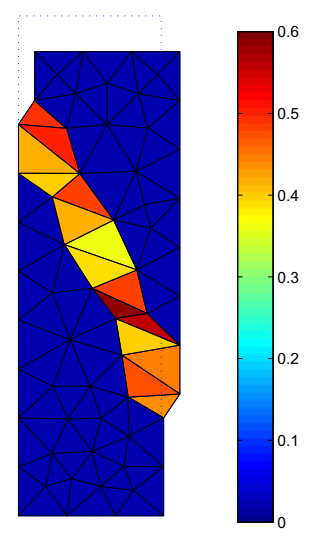

Fig. 17. Deviatoric strain contour at 7\% global axial strain for mesh 3.
To show that the model can capture the deformation modes observed in the experiment, we compare the computed local lateral strain with the experiment data, as shown in Fig. 15. "Upper" or "lower" means that the lateral strain was computed/measured above or below the localization band. From both simulation and experiment results, it can be seen that the mechanical behavior in the "upper" and "lower" block differ significantly. Once localization occurs, the upper part of the sample will slide, inducing large amount of lateral strain, while the lower part remains almost rigid and intact. Again, four meshes give identical results.

Remark 5. It should be pointed out that the strain level is higher than typical small strain assumption. However, in the experiment, the strains are actually computed by using small strain calculations. Therefore, the small strain formulation in the AES is consistent with the experiment. Also, the results by multiscale AES are not affected as much and match well with experiment data. Extension of the method to finite strains is currently under development.

The deformed meshes at $7 \%$ global axial strain are plotted in Fig. 16. The modes of deformation for all meshes are practically identical and all meshes propagate successfully to the end of the simulation, demonstrating the applicability of the framework to incorporate experimental data directly. Finally, the deviatoric strain contour at $7 \%$ global axial strain is plotted for mesh 3 . As expected, it is clear that the strains are concentrated in the localization band. The sample essentially unloads elastically outside the localization band. (see Fig. 17)

\section{Conclusions}

We have presented a reformulated AES method within a hierarchical multiscale framework for modeling localization behavior in granular materials. A key feature of this framework is that, instead of constant or phenomenologically varied material properties typically used in standard AES methods, evolutions of material properties are extracted from grain scale computations or experimental data and used as direct input for the underlying continuum model. More importantly, the softening modulus, which appears in standard AES formulations, is bypassed. The stress-point integration algorithm is similar to the classical elastic-perfectly plastic model, resulting in a simple yet powerful method. Numerical examples of two plane strain compression tests, coupling the AES method with DEM and experimental data, demonstrate the applicability of this method. The mesh objectivity and numerical efficiency of this method are also shown in the numerical examples.

\section{Acknowledgments}

Support for this work is provided by AFOSR Grant No. FA955008-1-1092. This support is gratefully acknowledged. The DEM code used in this research is developed by Prof. Kuhn from University of Portland. The authors are grateful to Prof. Kuhn for making the source code available.

\section{References}

[1] A. Tordesillas, J. Shi, Micromechanical analysis of failure propagation in frictional granular materials, Int. J. Numer. Anal. Methods Geomech. 33 (2009) 1737-1768.

[2] R. Wan, P. Guo, M. Al-Mamun, Behavior of granular materials in relation to their fabric dependencies, Soils Found. 45 (2005) 77-86.

[3] F. Darve, L. Sibille, A. Daouadji, F. Nicot, Bifurcations in granular media: Macroand micro-mechanics approaches, C.R. Mec. 335 (2007) 496-515.

[4] F. Nicot, L. Sibille, F. Darve, Bifurcation in granular materials: An attempt for a unified framework, Int. J. Solids Struct. 46 (2009) 3938-3947. 
[5] J.W. Rudnicki, J.R. Rice, Conditions for localization of deformation in pressuresensitive dilatant materials, J. Mech. Phys. Solids 23 (1975) 371-394.

[6] K. Runesson, N.S. Ottosen, D. Peric, Discontinuous bifurcations of elasticplastic solutions at plane stress and plane strain, Int. J. Plast. 7 (1991) 99121.

[7] D. Bigoni, T. Hueckel, Uniqueness and localization. I: Associative and nonassociative elastoplasticity, Int. J. Solids Struct. 28 (1991) 197-213.

[8] R.I. Borja, J.E. Andrade, Critical state plasticity. Part VI: Meso-scale finite element simulation of strain localization in discrete granular materials, Comput. Methods Appl. Mech. Engrg. 195 (2006) 5115-5140.

[9] J.E. Andrade, R.I. Borja, Modeling deformation banding in dense and loose fluidsaturated sands, Finite Elem. Anal. Des. 43 (2007) 361-383.

[10] J.C. Simo, J. Oliver, F. Armero, An analysis of strong discontinuities induced by strain-softening in rate-independent inelastic solids, Comput. Mech. 12 (1993) 277-296.

[11] Z.P. Bažant, T. Belytschko, T.P. Chang, Continuum theory for strain-softening, J. Engrg. Mech., ASCE 110 (1984) 1666-1692.

[12] T. Belytschko, W.K. Liu, B. Moran, Nonlinear Finite Elements for Continua and Structures, John Wiley \& Sons Ltd., West Sussex, UK, 2000.

[13] T. Belytschko, J. Fish, B.E. Engelmann, A finite element with embedded localization zones, Int. J. Numer. Methods Engrg. 70 (1988) 59-89.

[14] E. Samaniego, T. Belytschko, Continuum-discontinuum modelling of shear bands, Int. J. Numer. Methods Engrg. 62 (2005) 1857-1872.

[15] T. Rabczuk, E. Samaniego, Discontinuous modelling of shear bands using adaptive meshfree methods, Comput. Methods Appl. Mech. Engrg. 197 (2008) 641-658.

[16] J. Oliver, Modelling strong discontinuities in solid mechanics via strain softening constitutive equations. Part 1: Fundamentals. Part 2: Numerical simulations, Int. J. Numer. Methods Engrg. 39 (1996) 3575-3623.

[17] J. Oliver, A.E. Huespe, E. Samaniego, E.W.V. Chaves, Continuum approach to the numerical simulation of material failure in concrete, Int. J. Numer. Anal. Methods Geomech. 28 (2004) 609-632.

[18] N. Moës, J. Bolbow, T. Belytschko, A finite element method for crack growth without remeshing, Int. J. Numer. Methods Engrg. 46 (1999) 131-150.

[19] J.M. Melenk, I. Babuška, The partition of unity finite element method: Basic theory and applications, Comput. Methods Appl. Mech. Engrg. 139 (1996) 289-314.

[20] F. Armero, K. Garikipati, An analysis of strong discontinuities in multiplicative finite strain plasticity and their relation with the numerical simulation of strain localization in solids, Int. J. Solids Struct. 33 (1996) 2863-2885.

[21] C. Callari, F. Armero, Finite element methods for the analysis of strong discontinuities in coupled poro-plastic media, Comput. Methods Appl. Mech. Engrg. 191 (2002) 4371-4400.

[22] M. Jirásek, Comparative study on finite elements with embedded discontinuities, Comput. Methods Appl. Mech. Engrg. 188 (2000) 307-330.

[23] J. Oliver, A.E. Huespe, E. Samaniego, A study on finite elements for capturing strong discontinuities, Int. J. Numer. Methods Engrg. 56 (2003) 2134-2161.

[24] J. Oliver, A comparative study on finite elements for capturing strong discontinuities e-fem vs x-fem, Comput. Methods Appl. Mech. Engrg. 195 (2006) 4732-4752.

[25] J.C. Simo, M.S. Rifai, A class of mixed assumed strain methods and the method of incompatible modes, Int. J. Numer. Methods Engrg. 29 (1990) 1595-1638.

[26] J.C. Simo, J. Oliver, A new approach to the analysis and simulation of strain softening in solids, in: Z.P. Bazant, Z. Bittnar, M. Jirasek, J. Mazars (Eds.), Fracture and Damage in Quasibrittle Structures, E \& FN Spon, London, 1994, pp. 25-39.

[27] Richard A. Regueiro, Ronaldo I. Borja, A finite element model of localized deformation in frictional materials taking a strong discontinuity approach, Finite Elem. Anal. Des. 33 (1999) 283-315.

[28] Richard A. Regueiro, Ronaldo I. Borja, Plane strain finite element analysis of pressure sensitive plasticity with strong discontinuity, Int. J. Solids Struct. 38 (2001) 3647-3672.

[29] Ronaldo I. Borja, Richard A. Regueiro, Strain localization in frictional materials exhibiting displacement jumps, Comput. Methods Appl. Mech. Engrg. 190 (2001) 2555-2580

[30] Ronaldo I. Borja, A finite element model for strain localization analysis of strongly discontinuous fields based on standard Galerkin approximation, Comput. Methods Appl. Mech. Engrg. 190 (2000) 1529-1549.
[31] Ronaldo I. Borja, Assumed enhanced strain and the extended finite element methods: A unification of concepts, Comput. Methods Appl. Mech. Engrg. 197 (2008) 2789-2803.

[32] J. Mosler, G. Meschke, 3d Modelling of strong discontinuities in elastoplastic solids: Fixed and rotating localization formulations, Int. J. Numer. Methods Engrg. 57 (2003) 1553-1576.

[33] J. Mosler, A novel algorithmic framework for the numerical implementation of locally embedded strong discontinuities, Comput. Methods Appl. Mech. Engrg. 194 (2005) 4731-4757.

[34] J. Mosler, Modeling strong discontinuities at finite strains - a novel numerical implementation, Comput. Methods Appl. Mech. Engrg. 195 (2006) 4396-4419.

[35] C.D. Foster, R.I. Borja, R.A. Regueiro, Embedded strong discontinuity finite elements for fractured geomaterials with variable friction, Int. J. Numer. Methods Engrg. 72 (2007) 549-581.

[36] Q. Chen, A.E. Seifried, J.E. Andrade, J.W. Baker, Characterization of random fields and their impact on the mechanics of geosystems at multiple scales, Int. J. Numer. Anal. Methods Geomech. (2010), in press, doi:10.1002/nag.999.

[37] P.A. Cundall, O.D.L. Strack, A discrete numerical model for granular assemblies, Géotechnique 29 (1979) 47-65.

[38] J.P. Bardet, J. Proubet, A numerical investigation of the structure of persistent shear bands in granular media, Géotechnique 41 (1991) 599-613.

[39] J.P. Bardet, J. Proubet, Shear-band analysis in idealized granular material, J. Engrg. Mech. 118 (1992) 397-415.

[40] K. Iwashita, M. Oda, Rolling resistance at contacts in simulation of shear band development by dem, J. Engrg. Mech. 124 (1998) 285-292.

[41] K. Iwashita, M. Oda, Micro-deformation mechanism of shear banding process based on modified distinct element method, Powder Technol. 109 (2000) 192205.

[42] N. Hu, J.F. Molinari, Shear bands in dense metallic granular materials, J. Mech. Phys. Solids 52 (2004) 499-531.

[43] J.E. Andrade, X. Tu, Multiscale framework for behavior prediction in granular media, Mech. Mater. 41 (6) (2009) 652-669.

[44] J.E. Andrade, X. Tu, O. Chen, Return mapping for nonsmooth and multiscale elastoplasticity, Comput. Methods Appl. Mech. Engrg. 198 (2009) 2286-2296.

[45] J.E. Andrade, C.F. Avila, S.A. Hall, N. Lenoir, G. Viggiani, Multiscale modeling and characterization of granular matter: From grain kinematics to continuum mechanics, J. Mech. Phys. Solids 59 (2010) 237-250.

[46] M. Xu, Multiscale Methods: Bridging the Scales in Science and Engineering, Chapter: Concurrent Coupling of Atomistic and Continuum Models, Oxford University Press, 2009.

[47] C. Linder, F. Armero, Finite elements with embedded strong discontinuities for the modeling of failure in solids, Int. J. Numer. Methods Engrg. 72 (2007) 1391-1433.

[48] F. Armero, C. Linder, New finite elements with embedded strong discontinuities in the finite deformation range, Comput. Methods Appl. Mech. Engrg. 197 (2008) 3138-3170.

[49] J.E. Andrade, R.I. Borja, Capturing strain localization in dense sands with random density, Int. J. Numer. Methods Engrg. 67 (2006) 1531-1564.

[50] J. Christoffersen, M.M. Mehrabadi, S. Nemat-Nasser, A micromechanica description of granular material behavior, J. Appl. Mech. 48 (1981) 339-344.

[51] G.A. D'Addetta, E. Ramm, S. Diebels, W. Ehlers, A particle center based homogenization strategy for granular assemblies, Engrg. Comput. 21 (2004) 360-383.

52] C. Wellmann, C. Lillie, P. Wriggers, Homogenization of granular materia modeled by a three-dimensional discrete element method, Comput. Geotech. 35 (2008) 394-405.

[53] M.A. Mooney, R.J. Finno, M.G. Viggiani, A unique critical state for sand?, Geotech. Geoenviron. Engrg. 124 (1998) 1100-1108.

[54] S.A. Hall, M. Bornert, J. Desrues, Y. Pannier, N. Lenoir, G. Viggiani, P. Bésuelle Discrete and continuum analysis of localized deformation in sand using x-ray micro ct and volumetric digital image correlation, Géotechnique 60 (2010) 315-322.

[55] N. Lenoir, M. Bornert, J. Desrues, G. Viggiani, 3d digital image correlation applied to $\mathrm{x}$-ray microtomography images from triaxial compression tests on argillaceous rock, Strain 43 (2007) 193-205.

[56] D.M. Wood, Soil Behaviour and Critical State Soil Mechanics, Cambridge University Press, Cambridge, UK, 1990.

[57] I. Vardoulakis, Shear band inclination and shear modulus of sand in biaxial tests, Int. J. Numer. Anal. Methods Geomech. 4 (1980) 103-119. 